\title{
Testes de vigor em sementes de alface
}

\author{
Ana Lúcia P Kikuti'; Julio Marcos Filho² \\ ${ }^{1}$ UEMS-UUC, 79540-000 Cassilândia-MS; alkikuti@uems.br; ${ }^{2}$ USP-ESALQ, Dep ${ }^{\text {to. }}$ Prod. Vegetal, C. Postal 09, 13418-900 Piracicaba- \\ SP; jmarcos@esalq.usp.br
}

\section{RESUMO}

O vigor da semente tem sido avaliado por meio de testes fisiológicos, bioquímicos e de tolerância a estresse. Mais recentemente, com o uso da análise computadorizada de imagens de plântulas, podem ser obtidas informações objetivas, em período relativamente curto, com menor interferência humana. $\mathrm{O}$ objetivo nesta pesquisa foi identificar testes eficientes para avaliação do vigor de sementes de alface, com ênfase para a análise de imagens digitais de plântulas. Sementes de 12 lotes (seis da cultivar Vanda e seis da Vera) foram avaliadas por meio de testes de germinação, germinação a baixa temperatura $\left(15^{\circ} \mathrm{C}\right)$, emergência de plântulas e envelhecimento acelerado tradicional e com solução salina $(\mathrm{NaCl} 40 \%)$ saturada, a $41^{\circ} \mathrm{C} / 48 \mathrm{~h}$ e $41{ }^{\circ} \mathrm{C} / 72 \mathrm{~h}$. Utilizou-se, também, o sistema de análise computadorizada de imagens de plântulas (SVIS ${ }^{\circledR}$ ), desenvolvido pela Ohio State University (EUA), que forneceu dados referentes ao índice de vigor, uniformidade e crescimento de plântulas. Os testes foram realizados a cada três meses, durante 12 meses de armazenamento, sob condições ambiente. Com base nos resultados obtidos, concluiu-se que os testes de envelhecimento acelerado com uso de solução saturada de $\mathrm{NaCl}$, a $41^{\circ} \mathrm{C}$ por 48 e 72 horas e de velocidade de germinação, e os parâmetros obtidos com o software SVIS, índice de crescimento de plântulas e comprimento da raiz primária, são eficientes na avaliação do vigor de lotes de sementes de alface.

Palavras-chave: Lactuca sativa, análise de imagens de plântulas, potencial fisiológico.

\begin{abstract}
\section{Seed vigor tests for lettuce seeds}

The seed vigor is evaluated by traditional tests and more recently by seedling imaging analysis to obtain objective information within a short period of time. The objective of the study was to verify the accuracy of accepted vigor tests in comparison to an automated computer imaging system for seedling evaluation $\left(\mathrm{SVIS}^{\circledR}\right)$ in order to assess lettuce seed vigor. Six seed lots each of two cultivars (Vanda and Vera) were evaluated in the germination, cold germination $\left(15^{\circ} \mathrm{C}\right)$, seedling emergence and accelerated aging (traditional and saturated salt at $41^{\circ} \mathrm{C} / 48 \mathrm{~h}$ and $41^{\circ} \mathrm{C} / 72 \mathrm{~h}$ ). The Seed Vigor Imaging System was also utilized, developed by the Ohio State University $\left(\right.$ SVIS $^{\circledR}$ ) that generate numerical values of seedling growth, uniformity and vigor index. The tests were carried out in five evaluation times during a 12-month storage period. The results indicated that saturated salt accelerated aging at $41^{\circ} \mathrm{C} / 48$ or 72 hours and the rate of germination test, are sensitive to detect differences in the physiological potential of lettuce seed lots as well as the seedling growth and root length parameters evaluated by SVIS.
\end{abstract}

Keywords: Lactuca sativa, seedling imaging analysis, physiological potential.

\section{(Recebido para publicação em 3 de maio de 2011; aceito em 2 de fevereiro de 2012) (Received on May 3, 2011; accepted on February 2, 2012)}

$\mathrm{O}^{\mathrm{s}}$ testes de vigor são ferramentas importantes para a complementação das informações obtidas no teste de germinação, apresentando relação mais estreita com o desempenho de sementes durante o armazenamento e em condições de campo. O conhecimento do potencial fisiológico das sementes permite a produção de mudas com tamanho e qualidade uniformes, com vantagens ao desenvolvimento das plantas, principalmente em espécies em que a condução da cultura comercial requer o transplante, como a alface.

A disponibilidade de informações sobre testes para estimar o nível de vigor de lotes de sementes de alface é relativamente limitada. Para o teste de envelhecimento acelerado, procedimento tradicional, Hampton \& TeKrony (1995) indicaram o uso da combinação $41^{\circ} \mathrm{C} / 72 \mathrm{~h}$ e, Pereira \& Nascimento
(2003), a combinação $41^{\circ} \mathrm{C} / 48 \mathrm{~h}$; e no teste de deterioração controlada Powell (1995) recomendou a combinação do teor de água de $20 \%, 45^{\circ} \mathrm{C}$ e 24 horas de incubação em banho-Maria.

Propostas para a determinação do vigor por meio de análise computadorizada de imagens de plântulas têm sido utilizadas, visando à obtenção de informações objetivas, em período relativamente curto e sem a interferência humana. Neste sentido, Geneve \& Kester (2001) obtiveram resultados promissores com o uso de análise de imagens em couve-flor, tomate, pimentão, impatiens, vinca e margarida, para avaliação do comprimento de plântulas.

Posteriormente, Sako et al. (2001) propuseram um método automatizado para avaliação do vigor de sementes de alface, por meio da captura de imagens de plântulas e determinações simultâneas do comprimento do hipocótilo, da raiz primária, da plântula inteira e da relação raiz/hipocótilo, em plântulas avaliadas aos três dias após a instalação do teste de germinação. A técnica envolve o processamento das imagens com o uso do software "Seed Vigor Imaging System ${ }^{\circledR}$ " (SVIS), desenvolvido na Ohio State University; o processamento de imagens de plântulas ou de suas partes permite estabelecer índices de vigor, uniformidade e crescimento das plântulas.

$\mathrm{O}$ "Seed Vigor Imaging System ${ }^{\circledR}$ " foi modificado e adaptado para avaliação do vigor de sementes de soja por Hoffmaster et al. (2003). O programa desenvolvido para soja foi utilizado com sucesso por Marcos Filho et al. (2006) para avaliação do vigor de sementes de melão. Outros sistemas de análise de imagens têm sido divulgados para avaliação do período necessário para 
a germinação de sementes de girassol (Ducournau et al., 2005) e do crescimento de plântulas de rabanete (Tohidloo \& Kruse, 2009).

Com base no exposto, o objetivo nesta foi comparar a eficiência de procedimentos já consagrados para outras espécies, comparados à técnica de análise de imagens digitais de plântulas, para a determinação do vigor de sementes de alface.

\section{MATERIAL E MÉTODOS}

A pesquisa foi conduzida no Laboratório de análise de imagens e no Laboratório de análise de sementes da ESALQ em Piracicaba (SP), de junho de 2007 a julho de 2008 .

Amostras de 12 lotes de sementes de alface, seis da cultivar Vanda e seis da Vera, foram homogeneizadas, embaladas em sacos de plástico aluminizado, hermeticamente fechados e armazenadas em ambiente de laboratório durante o período de condução do experimento.

Os dados mensais médios de temperatura $\left({ }^{\circ} \mathrm{C}\right)$ máxima, mínima e umidade relativa do ar do período de julho de 2007 a julho de 2008 foram: temperaturas máximas (médias mensais): 25,0; 28,7; 31,3; 32,4; 28,8; 30,8; 28,9; 30,7; 29,$7 ; 28,0 ; 24,7 ; 25,3 ; 26,7$; temperaturas mínimas (médias mensais): 10,9; 11,$6 ; 15,2 ; 17,3 ; 17,4 ; 18,8 ; 18,5 ; 18,2$; $17,1 ; 12,5 ; 12,6 ; 9,8)$ e umidade relativa do ar (\%, médias mensais): 87,$3 ; 69,0$; 57,0; 71,0; 82,0; 80,7; 87,6; 85,9; 81,9; $84,3 ; 78,0 ; 80,2 ; 66,5)$.

Foram realizados os testes descritos a seguir para avaliação do potencial fisiológico das sementes, em cinco épocas (antes e após 3, 6, 9 e 12 meses de armazenamento).

Velocidade e porcentagem de germinação - Oito repetições de 25 sementes para cada lote foram colocadas em caixas de plástico transparente (11 x 11 x $3 \mathrm{~cm}$ ), envolvidas por sacos de plástico transparente, sobre duas folhas de papel mata-borrão e umedecidas com quantidade de água equivalente a 2,5 vezes a massa do substrato seco. As caixas com as sementes foram colocadas em câmara de germinação tipo BOD a $20^{\circ} \mathrm{C}$, com luz constante. As avaliações foram realizadas do terceiro até o oitavo dia após a instalação do teste, computando-se o número médio de plântulas normais seguindo critérios estabelecidos nas Regras para Análise de Sementes (Brasil, 2009). O índice de velocidade de germinação foi calculado de acordo com a fórmula proposta por Maguire (1962). A porcentagem de germinação foi calculada de acordo com o número de plântulas normais obtidas sete dias após a instalação do teste.

Velocidade e porcentagem de germinação à baixa temperatura - Foi conduzido do mesmo modo descrito para o teste de germinação; porém em BOD a $15^{\circ} \mathrm{C}$ e, as avaliações, realizadas diariamente do sexto dia até o nono dia após a instalação do teste. $\mathrm{O}$ índice de velocidade de germinação foi calculado de acordo com a fórmula proposta por Maguire (1962). A porcentagem de germinação foi calculada de acordo com o número de plântulas normais obtidas oito dias após a instalação do teste.

Envelhecimento acelerado (procedimento tradicional) - Amostras de sementes pré pesadas (1,3 g) foram colocadas em tela de alumínio suspensa em caixa de plástico (11 x 11 x $3 \mathrm{~cm})$ contendo $40 \mathrm{~mL}$ de água. As amostras de sementes foram mantidas em incubadora a $41^{\circ} \mathrm{C}$, durante 48 e 72 horas. Decorrido cada período de envelhecimento, quatro amostras de 50 sementes por lote foram colocadas para germinar, a $20^{\circ} \mathrm{C}$, com luz constante. A avaliação foi realizada aos cinco dias após a semeadura, computando-se a porcentagem de plântulas normais.

Envelhecimento acelerado (solução saturada de $\mathbf{N a C l}$ ) - O teste foi realizado como descrito para o de envelhecimento acelerado tradicional, porém, foi colocado no interior de cada caixa de plástico $40 \mathrm{~mL}$ de solução saturada de cloreto de sódio (40 g de $\mathrm{NaCl}$ em $100 \mathrm{~mL}$ de água), visando proporcionar ambiente com $76 \%$ de umidade relativa do ar, de acordo com procedimento proposto por Jianhua \& McDonald (1997).

Velocidade e porcentagem de emergência de plântulas - Quatro repetições de 50 sementes foram distribuídas em células individuais de bandejas de poliestireno de 200 células, contendo substrato comercial plantmax ${ }^{\circledR}$. As bandejas foram mantidas em ambiente de laboratório, realizando-se irrigações diárias. As avaliações foram realizadas diariamente do $4^{\circ}$ até o $8^{-}$dia após a instalação do teste, para obtenção do índice de velocidade de emergência, que foi calculado com base na fórmula proposta por Maguire (1962). A avaliação da porcentagem de emergência foi realizada aos 14 dias após a instalação do teste. Para essas avaliações foram computadas as plântulas que apresentaram a parte aérea normal com tamanho igual ou superior a $1,0 \mathrm{~cm}$.

Determinação do teor de água Foi realizada antes do início dos testes e, após a incubação para o teste de envelhecimento acelerado, em todas as épocas de avaliação. Foi utilizado o método da estufa a $105^{\circ} \mathrm{C}$ por 24 horas, de acordo com as Regras para Análise de Sementes (Brasil, 2009). Os resultados foram expressos em porcentagem (base úmida) média para cada lote.

Avaliação do vigor por meio do software SVIS - As sementes foram colocadas para germinar em caixas de plástico transparente $(11,0 \times 11,0 \times 3,5$ $\mathrm{cm})$ contendo três folhas de papel mata-borrão azul, umedecidas com quantidade de água equivalente a 2,5 vezes a massa do substrato seco. As folhas de papel mata-borrão azul foram utilizadas para proporcionar contraste com as plântulas no momento da captura da imagem. As sementes foram distribuídas em duas fileiras, no terço superior do substrato, cobrindo-se em seguida com uma folha de papel mata borrão. Este procedimento se fez necessário para manutenção das sementes na posição original, pois as caixas foram dispostas em câmara de germinação tipo BOD formando um ângulo de $60^{\circ}$ a $70^{\circ}$ com a horizontal, para que o desenvolvimento das plântulas obedecesse ao geotropismo natural. As caixas de plástico foram envolvidas por sacos de plástico transparente e colocadas na $\mathrm{BOD}$, a $20^{\circ} \mathrm{C}$, durante três dias, no escuro.

As imagens de quatro repetições de 25 plântulas por lote, de cada vez, foram capturadas por scanner HP Scanjet 2004, operado por software Photosmart com resolução de 100 dpi. As imagens capturadas foram analisadas pelo 
software SVIS.

O programa gera valores de índice de vigor, uniformidade de desenvolvimento, crescimento de plântula, comprimento de hipocótilo e de raiz primária, conforme descrito por Sako et al. (2001).

Os valores de índice de vigor (0 a 1000) são baseados na rapidez e uniformidade de desenvolvimento das plântulas em relação ao máximo valor possível para plântulas de alface com três dias de idade. Os valores de uniformidade (0 a 1000) são baseados no desvio em relação ao desenvolvimento padrão das plântulas, programado no software. O comprimento médio de hipocótilo e raiz é calculado com base na leitura do comprimento destes, realizada pelo software.

Foi utilizado o delineamento inteiramente casualizado, com quatro repetições. A análise estatística dos dados obtidos nos diferentes testes foi realizada comparando-se os lotes dentro de cada cultivar, para cada época de avaliação e teste. Foi utilizado o programa SISVAR e, o teste de Tukey para a comparação das médias.

\section{RESULTADOS E DISCUSSÃO}

Os resultados obtidos na primeira, terceira e quinta épocas de avaliação, nos testes tradicionais para avaliação do potencial fisiológico da cultivar Vanda encontram-se na Tabela 1.

Houve redução do potencial fisioló- gico das sementes devido à deterioração natural, causada pela temperatura, ao longo do armazenamento, já que não houve grande variação no teor de água das sementes, devido à embalagem utilizada de sacos de plástico aluminizado, hermeticamente fechados.

Houve diferença estatística entre os dados da primeira, terceira e quinta épocas de avaliação, para as duas cultivares. Os dados obtidos na segunda época de avaliação foram semelhantes aos obtidos na primeira época e, os obtidos na quarta época foram semelhantes aos obtidos na quinta época.

Para a cultivar Vanda, observou-se no índice de vigor e comprimento de raiz avaliados por meio do software SVIS, assim como nos testes de germinação

Tabela 1. Velocidade e porcentagem de germinação (VG e G), velocidade e porcentagem de germinação a baixa temperatura (VGBT e GBT), velocidade e porcentagem de emergência de plântulas (VE e E), envelhecimento acelerado tradicional (EAT) e com solução saturada de sal (EASS) de seis lotes de alface cultivar Vanda, obtidos na 1a época de avaliação (antes do armazenamento), 3 a (após seis meses) e 5a (após 12 meses) (germination speed and percentage (VG and G), low temperature germination speed and percentage (VGBT and GBT), seedling speed and percentage (VE and E), traditional accelerated ageing (EAT) and with saturated salt solution (EASS) of six lots of lettuce cultivar Vanda, obtained on $1^{\text {st }}$ evaluation time (before storage), $3^{\text {rd }}$ (six months later) and $5^{\text {th }}$ (12 months later)). Piracicaba, ESALQ, 2008.

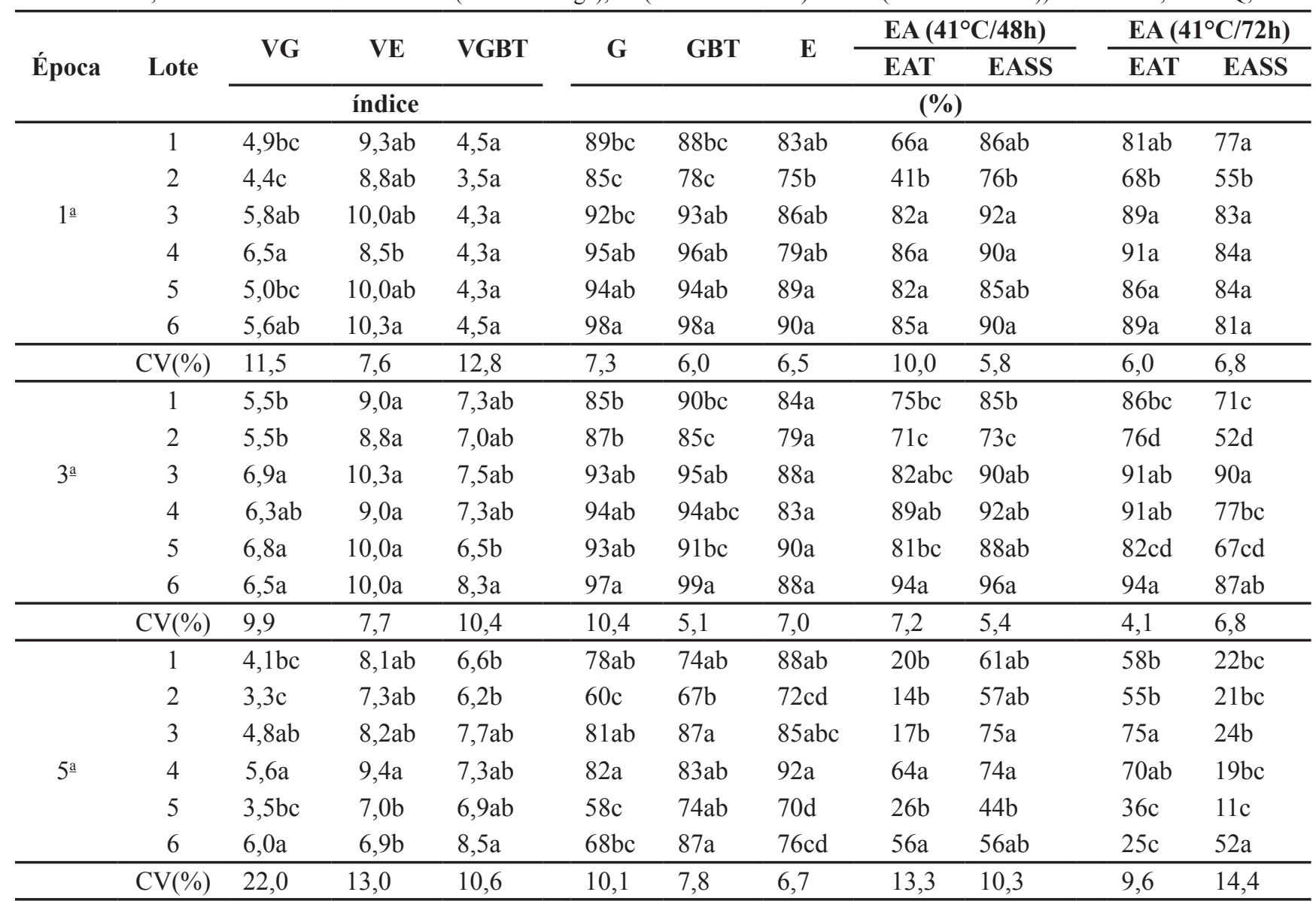

Comparações entre lotes, dentro de cada teste, para cada época separadamente (Tukey, $\mathrm{p} \leq 0,05)$ (comparisons between lots, for each test and each time separately by Tukey test, $\mathrm{p} \leq 0,05)$. 
Tabela 2. Velocidade e porcentagem de germinação (VG e G), velocidade e porcentagem de germinação a baixa temperatura (VGBT e GBT), velocidade e porcentagem de emergência de plântulas (VE e E), envelhecimento acelerado tradicional (EAT) e com solução saturada de sal (EASS) de seis lotes de alface cultivar Vera, obtidos na $1^{\text {a }}$ época de avaliação (antes do armazenamento), $3^{3}$ (após seis meses) e $5^{\text {a }}$ (após 12 meses) (germination speed and percentage (VG and G), low temperature germination speed and percentage (VGBT and GBT), seedling speed and percentage (VE and E), traditional accelerated ageing (EAT) and with saturated salt solution (EASS) of six lots of lettuce cultivar Vera, obtained on $1^{\text {st }}$ evaluation time (before storage), $3^{\text {rd }}$ (six months later) and $5^{\text {th }}$ (12 months later)). Piracicaba, ESALQ, 2008.

\begin{tabular}{|c|c|c|c|c|c|c|c|c|c|c|c|}
\hline \multirow{3}{*}{ Época } & \multirow{3}{*}{ Lotes } & \multirow{2}{*}{ VG } & \multirow{2}{*}{ VE } & \multirow{2}{*}{ VG BT } & \multirow{2}{*}{ G } & \multirow{2}{*}{ G BT } & \multirow{2}{*}{$\mathbf{E}$} & \multicolumn{2}{|c|}{$\mathrm{EA}\left(41^{\circ} \mathrm{C} / 48 \mathrm{~h}\right)$} & \multicolumn{2}{|c|}{$\operatorname{EA}\left(41^{\circ} \mathrm{C} / 72 \mathrm{~h}\right)$} \\
\hline & & & & & & & & EAT & EASS & EAT & EASS \\
\hline & & \multicolumn{3}{|c|}{ índice } & \multicolumn{7}{|c|}{$(\%)$} \\
\hline \multirow{6}{*}{1} & 7 & $5,0 \mathrm{c}$ & $9,4 \mathrm{a}$ & $5,1 \mathrm{a}$ & $98 \mathrm{a}$ & $98 \mathrm{a}$ & $94 a$ & $28 b$ & $89 a$ & $25 \mathrm{c}$ & $90 a$ \\
\hline & 8 & $7,4 \mathrm{a}$ & $9,4 \mathrm{a}$ & $5,4 \mathrm{a}$ & $100 \mathrm{a}$ & $99 a$ & $84 b$ & $86 a$ & $91 \mathrm{a}$ & $92 \mathrm{a}$ & $92 a$ \\
\hline & 9 & $6,5 b$ & $10,4 \mathrm{a}$ & $5,3 \mathrm{a}$ & $99 \mathrm{a}$ & $100 \mathrm{a}$ & $70 \mathrm{c}$ & $88 \mathrm{a}$ & $89 a$ & $85 a$ & $92 \mathrm{a}$ \\
\hline & 10 & $6,8 \mathrm{ab}$ & $10,1 \mathrm{a}$ & $5,4 \mathrm{a}$ & $100 \mathrm{a}$ & $98 \mathrm{a}$ & $84 b$ & $96 a$ & $95 \mathrm{a}$ & $91 \mathrm{a}$ & $91 \mathrm{a}$ \\
\hline & 11 & $7,0 \mathrm{ab}$ & $10,3 \mathrm{a}$ & $5,2 \mathrm{a}$ & $99 \mathrm{a}$ & $99 a$ & $97 \mathrm{a}$ & $46 \mathrm{~b}$ & $94 \mathrm{a}$ & $54 \mathrm{~b}$ & $94 a$ \\
\hline & 12 & $7,3 \mathrm{a}$ & $9,1 \mathrm{a}$ & $5,4 \mathrm{a}$ & $99 \mathrm{a}$ & $99 \mathrm{a}$ & $91 \mathrm{ab}$ & $92 \mathrm{a}$ & $94 \mathrm{a}$ & $84 a$ & $91 \mathrm{a}$ \\
\hline & $\mathrm{CV}(\%)$ & 11,5 & 7,6 & 12,8 & 7,3 & 6,0 & 6,5 & 10,0 & 5,8 & 6,8 & 6,0 \\
\hline \multirow{6}{*}{$3^{\mathrm{a}}$} & 7 & $5,0 \mathrm{c}$ & $10,0 \mathrm{ab}$ & $7,1 \mathrm{a}$ & $96 a$ & $98 \mathrm{a}$ & $91 \mathrm{a}$ & $30 \mathrm{c}$ & $84 c$ & $43 c$ & $87 b$ \\
\hline & 8 & $7,4 \mathrm{a}$ & $9,0 \mathrm{c}$ & $8,0 \mathrm{a}$ & $99 \mathrm{a}$ & $99 a$ & $75 \mathrm{c}$ & $93 a$ & $99 \mathrm{a}$ & $56 \mathrm{bc}$ & $95 \mathrm{ab}$ \\
\hline & 9 & $7,3 \mathrm{a}$ & $7,6 \mathrm{~d}$ & $8,2 \mathrm{a}$ & $98 \mathrm{a}$ & $99 a$ & $62 d$ & $92 \mathrm{a}$ & $93 a b c$ & $62 \mathrm{bc}$ & $97 \mathrm{ab}$ \\
\hline & 10 & $6,6 \mathrm{ab}$ & $9,6 \mathrm{bc}$ & $7,7 \mathrm{a}$ & $94 a$ & $98 \mathrm{a}$ & $82 \mathrm{bc}$ & $93 a$ & $97 \mathrm{ab}$ & $92 \mathrm{a}$ & $99 \mathrm{a}$ \\
\hline & 11 & $6,1 \mathrm{~b}$ & $9,3 \mathrm{abc}$ & $7,6 \mathrm{a}$ & $99 \mathrm{a}$ & $99 a$ & $83 \mathrm{abc}$ & $71 \mathrm{~b}$ & $92 \mathrm{bc}$ & $72 b$ & $96 a b$ \\
\hline & 12 & 6,9ab & $11,1 \mathrm{a}$ & $7,7 \mathrm{a}$ & $99 \mathrm{a}$ & $99 \mathrm{a}$ & $90 \mathrm{ab}$ & $90 \mathrm{a}$ & $96 a b$ & $89 \mathrm{a}$ & $93 \mathrm{ab}$ \\
\hline & $\mathrm{CV}(\%)$ & 8,5 & 6,1 & 8,9 & 9,6 & 7,1 & 4,9 & 5,4 & 5,8 & 8,7 & 6,7 \\
\hline \multirow{7}{*}{$5 \mathrm{a}$} & 7 & $1,7 b$ & $4,5 b$ & $3,6 c$ & $25 b$ & $46 \mathrm{~b}$ & $49 \mathrm{~b}$ & $7 \mathrm{c}$ & $27 \mathrm{c}$ & $2 \mathrm{c}$ & $12 \mathrm{c}$ \\
\hline & 8 & $5,2 \mathrm{a}$ & $9,9 \mathrm{a}$ & $8,3 \mathrm{a}$ & $82 a$ & $94 a$ & $91 \mathrm{a}$ & $78 \mathrm{a}$ & $80 \mathrm{a}$ & $55 \mathrm{a}$ & $74 a$ \\
\hline & 9 & $5,5 \mathrm{a}$ & $9,5 \mathrm{a}$ & $7,8 \mathrm{ab}$ & $80 \mathrm{a}$ & $93 a$ & $91 \mathrm{a}$ & $66 a b$ & $81 \mathrm{a}$ & $1 \mathrm{c}$ & $70 \mathrm{a}$ \\
\hline & 10 & $5,5 \mathrm{a}$ & $8,9 \mathrm{a}$ & $7,8 \mathrm{ab}$ & $81 \mathrm{a}$ & $93 a$ & $87 \mathrm{a}$ & $71 \mathrm{a}$ & $75 a$ & $3 c$ & 71a \\
\hline & 11 & $1,8 b$ & $3,5 \mathrm{~b}$ & $2,7 \mathrm{c}$ & $28 b$ & $34 \mathrm{~b}$ & $36 \mathrm{~b}$ & $5 c$ & $16 \mathrm{c}$ & $0 \mathrm{c}$ & $14 \mathrm{c}$ \\
\hline & 12 & $4,9 \mathrm{a}$ & $8,8 \mathrm{a}$ & $6,8 b$ & $80 \mathrm{a}$ & $86 a$ & $87 \mathrm{a}$ & $46 \mathrm{~b}$ & $61 \mathrm{~b}$ & $28 \mathrm{~b}$ & $45 \mathrm{~b}$ \\
\hline & $\mathrm{CV}(\%)$ & 15,9 & 8,4 & 10,0 & 11,5 & 8,7 & 6,6 & 13,5 & 7,0 & 34,1 & 14,6 \\
\hline
\end{tabular}

Comparações entre lotes, dentro de cada teste, para cada época separadamente (Tukey, $\mathrm{p} \leq 0,05)$ (comparisons between lots, for each test and each time separately by Tukey test, $\mathrm{p} \leq 0,05$ ).

(velocidade e porcentagem), a baixa temperatura e envelhecimento acelerado em todos os procedimentos, que em geral, o lote 2 proporcionou valores inferiores aos dos demais lotes (Tabelas 1 e 3). Verificou-se, de maneira geral, que os lotes 4 e 6 apresentaram maior nível de vigor em relação aos demais. No entanto, após 12 meses de armazenamento houve maior redução do potencial fisiológico do lote 6 em relação ao lote 4. A discriminação dos lotes de pior e de melhor desempenho obtida com o uso do SVIS, de maneira semelhante com aquela obtida em testes tradicionais, também foi observada por Marcos Filho et al. (2006), em sementes de melão.

A eficiência do programa "Seed Vigor Imaging System ${ }^{\circledR}$ " (SVIS) tam- bém foi relatada por Sako et al. (2001) para avaliação do vigor de sementes de alface, Hoffmaster (2003), Sekharan (2005) e Marcos Filho et al. (2009) para sementes de soja e Otoni \& McDonald (2005) para sementes de milho. Cabe ressaltar que, embora Sako et al. (2001) tenham relatado a eficiência do software para avaliação do vigor de sementes de alface, os resultados obtidos não foram comparados aos obtidos em diferentes testes tradicionais.

Para a cultivar Vanda, a avaliação do comprimento de raiz por meio do software, em todas as épocas de avaliação (Tabela 3), proporcionou informações semelhantes à da quinta época de avaliação, quando considerados os testes de germinação e de emergência de plân- tulas (Tabela 1). O nível de vigor das sementes, na quinta época de avaliação, já havia diminuído naturalmente em razão do armazenamento ter sido realizado em condições não controladas, indicando a acuidade da característica comprimento de raiz avaliada por meio do software para avaliação do vigor de sementes de alface. Esses resultados corroboram com os de Guimarães et al. (1993) e Nascimento \& Pereira (2007), os quais indicaram a eficiência da avaliação do comprimento de raiz primária, realizado manualmente no teste de comprimento de plântulas, para separar lotes de alface em diferentes níveis de vigor.

De acordo com Nakagawa et al. (1999), os testes que avaliam o crescimento de plântulas são os mais sensíveis 
Tabela 3. Índice de vigor (V), crescimento (C), uniformidade (U), comprimento de hipocótilo (CH) e de raiz (CR) de plântulas de alface determinados em análises SVIS, de seis lotes de alface cultivar Vanda e seis do Vera, obtidos na 1a época de avaliação (antes do armazenamento), 3a (após seis meses) e $5^{\text {a }}$ (após 12 meses) (vigor index (V), uniformity (U), growth (C) and length of hypocotyls (CH) and roots (CR) of lettuce seedlings determinated in analysis SVIS of six lettuce lots of cultivar Vanda and Vera, obtained on $1^{\mathrm{st}}$ evaluation time (before storage), $3^{\text {rd }}$ (six months later) and $5^{\text {th }}$ (12 months later)). Piracicaba, ESALQ, 2008.

\begin{tabular}{|c|c|c|c|c|c|c|c|c|c|c|c|c|}
\hline \multirow{3}{*}{$\begin{array}{c}\text { Épo- } \\
\text { ca }\end{array}$} & \multicolumn{6}{|c|}{ Cultivar Vanda } & \multicolumn{6}{|c|}{ Cultivar Vera } \\
\hline & Lote & $\mathbf{V}$ & C & $\mathbf{U}$ & $\mathbf{C H}$ & CR & Lote & V & $\mathbf{C}$ & $\mathbf{U}$ & $\mathbf{C H}$ & CR \\
\hline & & \multicolumn{3}{|c|}{ índice } & \multicolumn{2}{|c|}{$\mathbf{m m}$} & \multicolumn{4}{|c|}{ índice } & \multicolumn{2}{|c|}{$\mathbf{m m}$} \\
\hline \multirow{6}{*}{$1 \underline{a}$} & 1 & $239 a$ & $193 a$ & $349 a b$ & $19,0 \mathrm{a}$ & $37,8 \mathrm{a}$ & 7 & $198 b$ & $149 b$ & $315 b$ & $14,1 \mathrm{a}$ & $31,6 \mathrm{c}$ \\
\hline & 2 & $163 b$ & $115 b$ & $275 b$ & $11,0 \mathrm{a}$ & $22,8 \mathrm{c}$ & 8 & $267 \mathrm{a}$ & $213 a$ & $390 \mathrm{a}$ & $21,1 \mathrm{a}$ & $43,1 \mathrm{a}$ \\
\hline & 3 & $232 a$ & $190 \mathrm{a}$ & $332 \mathrm{ab}$ & $20,0 \mathrm{a}$ & $35,5 \mathrm{ab}$ & 9 & $212 b$ & $155 b$ & $348 \mathrm{ab}$ & $14,4 \mathrm{a}$ & $33,5 \mathrm{bc}$ \\
\hline & 4 & $205 \mathrm{ab}$ & $149 \mathrm{ab}$ & $337 \mathrm{ab}$ & $14,5 \mathrm{a}$ & $19,2 \mathrm{bc}$ & 10 & $238 \mathrm{ab}$ & $178 \mathrm{ab}$ & $380 \mathrm{a}$ & $16,8 \mathrm{a}$ & $37,7 b$ \\
\hline & 5 & $245 \mathrm{a}$ & $190 \mathrm{a}$ & $374 a$ & $19,3 \mathrm{a}$ & $36,3 \mathrm{ab}$ & 11 & $227 \mathrm{ab}$ & $166 \mathrm{~b}$ & $370 \mathrm{ab}$ & $16,7 \mathrm{a}$ & $33,4 \mathrm{bc}$ \\
\hline & 6 & $235 \mathrm{a}$ & $176 \mathrm{a}$ & $375 \mathrm{a}$ & $19,0 \mathrm{a}$ & $31,3 \mathrm{ab}$ & 12 & $265 a$ & $211 \mathrm{a}$ & $392 a$ & $20,5 \mathrm{a}$ & $43,6 a$ \\
\hline \multicolumn{2}{|c|}{ CV (\%) } & 12,6 & 15,3 & 11,5 & 23,6 & 11,7 & & 7,7 & 11,0 & 7,2 & 18,2 & 6,1 \\
\hline \multirow{6}{*}{$3^{\mathrm{a}}$} & 1 & $241 \mathrm{a}$ & $193 a$ & $356 a$ & $19,3 \mathrm{a}$ & $37,8 \mathrm{ab}$ & 7 & $157 b$ & $127 b c$ & $226 a$ & $11,1 b$ & $29,0 \mathrm{bc}$ \\
\hline & 2 & $142 \mathrm{c}$ & $101 b$ & $188 b$ & $7,5 b$ & $24,0 \mathrm{~d}$ & 8 & $234 \mathrm{a}$ & $182 \mathrm{ab}$ & $357 \mathrm{a}$ & $15,2 \mathrm{ab}$ & $42,5 \mathrm{a}$ \\
\hline & 3 & $179 \mathrm{bc}$ & $116 \mathrm{~b}$ & $328 \mathrm{a}$ & $9,5 b$ & $26,8 \mathrm{~cd}$ & 9 & $187 \mathrm{ab}$ & $129 b c$ & $324 a$ & $11,6 a b$ & $28,5 \mathrm{c}$ \\
\hline & 4 & $218 \mathrm{ab}$ & $176 a$ & $318 \mathrm{a}$ & $12,0 \mathrm{~b}$ & $44,8 \mathrm{a}$ & 10 & $237 \mathrm{a}$ & $194 a$ & $338 \mathrm{a}$ & $18,7 \mathrm{a}$ & $40,5 \mathrm{ab}$ \\
\hline & 5 & $201 \mathrm{ab}$ & $136 \mathrm{~b}$ & $354 \mathrm{a}$ & $10,3 b$ & $32,8 b c$ & 11 & $154 \mathrm{~b}$ & $121 b c$ & $231 \mathrm{a}$ & $11,9 \mathrm{ab}$ & $24,8 \mathrm{c}$ \\
\hline & 6 & $186 \mathrm{~b}$ & $112 b$ & $360 a$ & $9,0 \mathrm{~b}$ & $25,5 \mathrm{~cd}$ & 12 & $173 \mathrm{ab}$ & $120 \mathrm{c}$ & $296 a$ & $10,2 b$ & $27,9 \mathrm{c}$ \\
\hline \multicolumn{2}{|c|}{ CV (\%) } & 9,4 & 12,3 & 17,7 & 19,4 & 11,4 & & 18,0 & 18,7 & 20,6 & 24,6 & 16,6 \\
\hline \multirow{6}{*}{5} & 1 & $116 b c$ & $81 \mathrm{~cd}$ & $199 \mathrm{~cd}$ & $8,5 \mathrm{ab}$ & $15,8 b c$ & 7 & $95 b$ & $85 b$ & $119 b$ & $6,6 b$ & $18,0 \mathrm{~b}$ \\
\hline & 2 & $82 c$ & $60 \mathrm{~d}$ & $134 d$ & $5,5 \mathrm{~b}$ & $12,9 \mathrm{c}$ & 8 & $236 a$ & $204 a$ & $311 \mathrm{a}$ & $7,8 \mathrm{ab}$ & $21,9 \mathrm{ab}$ \\
\hline & 3 & $182 \mathrm{a}$ & $125 \mathrm{ab}$ & $317 \mathrm{a}$ & $12,9 \mathrm{a}$ & $24,2 \mathrm{a}$ & 9 & $219 a$ & $187 \mathrm{a}$ & $294 \mathrm{a}$ & $14,2 \mathrm{a}$ & $30,8 \mathrm{a}$ \\
\hline & 4 & $160 \mathrm{ab}$ & $108 \mathrm{abc}$ & $284 a b c$ & $11,2 \mathrm{ab}$ & $21,0 \mathrm{ab}$ & 10 & $236 a$ & $201 \mathrm{a}$ & $320 \mathrm{a}$ & $12,4 \mathrm{ab}$ & $26,7 \mathrm{ab}$ \\
\hline & 5 & $122 b c$ & $86 \mathrm{~cd}$ & $207 \mathrm{bcd}$ & $8,9 b$ & $16,6 b c$ & 11 & $74 \mathrm{~b}$ & $78 b$ & $64 b$ & $8,4 a b$ & $20,5 \mathrm{ab}$ \\
\hline & 6 & $180 \mathrm{a}$ & $129 a$ & $302 \mathrm{ab}$ & $12,9 \mathrm{a}$ & $25,9 \mathrm{a}$ & 12 & $225 a$ & $201 \mathrm{a}$ & $282 a$ & $9,5 \mathrm{ab}$ & $25,8 \mathrm{ab}$ \\
\hline \multicolumn{2}{|c|}{ CV (\%) } & 16,5 & 18,1 & 17,8 & 25,5 & 13,9 & & 13,9 & 14,6 & 17,3 & 29,8 & 22,1 \\
\hline
\end{tabular}

Comparações entre lotes, dentro de cada teste, para cada época separadamente, pelo teste de Tukey, $\mathrm{p} \leq 0,05$ (comparisons between lots, for each test and each time separately by Tukey test, $\mathrm{p} \leq 0,05)$.

para a determinação do vigor de sementes de muitas espécies. Marcos Filho et al. (2009) afirmaram que o comprimento de plântulas é o parâmetro mais sensível do programa SVIS, para avaliação do vigor de sementes de soja.

$O$ teste de germinação a baixa temperatura apresentou sensibilidade na identificação dos lotes com menor nível de vigor, de maneira semelhante ao teste de germinação e de envelhecimento acelerado na primeira e na terceira época de avaliação (Tabela 1).
No teste de envelhecimento acelerado, na quinta época de avaliação, observou-se queda no poder germinativo das sementes, para a cultivar Vanda (Tabela 1). Nesse período, somente os lotes 3 e 4 mantiveram germinação dentro dos padrões estabelecidos para a comercialização (acima de $80 \%$ ). Portanto, os demais lotes provavelmente estavam com nível de vigor muito baixo e a exposição a temperaturas e umidade relativa do ar elevadas, durante o teste, provocaram a perda do poder germina- tivo desses.

O objetivo dos testes de vigor é a separação entre lotes comercializáveis, com porcentual de germinação situado dentro de padrões comerciais, dentre outras características. Tendo como base essa premissa, o teste de envelhecimento foi eficiente enquanto todos os lotes se encontravam dentro dos padrões comerciais, ou seja, na primeira e terceira épocas de avaliação (Tabela 1).

O teor de água das sementes, determinado em todas as épocas de 
avaliação antes da realização dos testes, variou de 6,2 a 7,2\%, considerando todos os lotes analisados e todas as épocas de avaliação, para a cultivar Vanda e de 5,9 a 7,9\% para a 'Vera'. A variação relativamente pequena indica que não houve interferência do teor de água das sementes nos resultados obtidos nos testes de vigor.

$\mathrm{Na}$ determinação do teor de água realizada logo após a incubação para o teste de envelhecimento acelerado com solução saturada de $\mathrm{NaCl}$, observou-se que a maior variação foi de $1,4 \%$ entre o menor $(9,1 \%)$ e o maior valor $(10,5 \%)$, para a cultivar Vanda e de 8,3 a 10,2\% para a 'Vera', considerando-se todas as épocas de avaliação e tempos de incubação. Porém, a variação foi maior quando foi utilizado o procedimento tradicional, onde a amplitude entre o maior e o menor valor foi de $11,7 \%$ (26,7 a $38,4 \%$ ), para a cultivar Vanda, e de até $10,3 \%(24,1$ a $34,4 \%)$ para a 'Vera'. Esses valores superaram o limite de 3 a $4 \%$ de variação no teor de água, entre os lotes, após o período de incubação do teste de envelhecimento acelerado, de acordo com o recomendado por Marcos Filho (1999).

Variações no teor de água maiores que o recomendado também foram observadas por Rodo \& Marcos Filho (2003) para sementes de cebola, Rodo et al. (2005) para sementes de cenoura e Fessel et al. (2005) para sementes de brócolis, após a incubação para o teste de envelhecimento acelerado tradicional. Isto indica que as condições do teste de envelhecimento acelerado tradicional para sementes relativamente pequenas não são as mais indicadas, podendo comprometer os resultados.

Para a cultivar Vera, o teste de germinação a baixa temperatura não apresentou a mesma eficiência para discriminar os lotes em diferentes níveis de vigor observada para a cultivar Vanda (Tabelas 2 e 3 ).

Os lotes 7 e 11 foram identificados como de menor nível de vigor que os demais, já na primeira época de avaliação, pelos testes de envelhecimento acelerado tradicional, índice de crescimento e comprimento de raiz obtidos com o SVIS (Tabelas 2 e 3). Estes parâmetros avaliados com o SVIS também identifi- caram o lote 9 , juntamente com o 7 e o 11 , como de menor vigor. No resultado do teste de emergência de plântulas, o lote 9 também apresentou o menor nível de vigor.

$\mathrm{Na}$ terceira e quinta épocas de avaliação, além dos testes mencionados, os resultados dos testes de velocidade de germinação e de envelhecimento acelerado com solução salina a $41^{\circ} \mathrm{C} / 48 \mathrm{~h}$ também identificaram os lotes 7 e 11 como os de pior nível de vigor (Tabela 2 ). Estes mesmos lotes foram os que apresentaram menor nível de vigor, indicado pelos resultados nos testes de germinação e emergência de plântulas, realizados após 12 meses de armazenamento, ou seja, na quinta época de avaliação.

De modo geral, o teste de germinação a baixa temperatura $\left(15^{\circ} \mathrm{C}\right)$ parece não ter proporcionado estresse o suficiente nas sementes de alface das cultivares utilizadas nesta pesquisa. Resultados semelhantes foram observados por Nascimento \& Pereira (2007), que não encontraram diferenças entre lotes no teste de germinação a $10^{\circ} \mathrm{C}$, para sementes de alface da cultivar Everglades.

A velocidade de germinação foi adequada para discriminar os lotes em diferentes níveis de vigor, em todas as épocas de avaliação, para as duas cultivares. Ficou comprovada, também, a eficiência do teste de envelhecimento acelerado para avaliação do vigor de sementes de alface, estando essa informação de acordo com resultados obtidos por Franzin (2004) e Nascimento \& Pereira (2007). Entretanto, o uso de solução saturada de $\mathrm{NaCl}$ é mais indicado que o procedimento tradicional, por não apresentar variação acentuada no teor de água após a incubação para o teste, o que poderia ocasionar incoerência nos resultados.

Destaca-se a eficiência das características crescimento e comprimento de raiz, obtidas com o uso do programa "Seed Vigor Imaging System ${ }^{\circledR} "$ (SVIS), para a avaliação do vigor de sementes de alface. Por apresentar pequena interferência humana, as características avaliadas por meio do software permitem mais facilmente a padronização dos testes de vigor permitindo resultados rápidos e reproduzíveis entre labora- tórios o que muito poderá auxiliar as empresas em seus programas de controle de qualidade.

Pelos resultados obtidos neste trabalho conclui-se que os testes de envelhecimento acelerado com uso de solução saturada de cloreto de sódio, a $41^{\circ} \mathrm{C}$ durante 48 e 72 horas e de velocidade de germinação, foram os mais eficientes dentre os procedimentos tradicionais para a avaliação do potencial fisiológico de sementes de alface. As características obtidas com o software SVIS, índice de crescimento de plântulas e comprimento da raiz primária, proporcionaram resultados semelhantes aos dos testes tradicionais sendo eficientes para avaliação do vigor de sementes de alface, com destaque para a rapidez na obtenção dos resultados, além da menor interferência humana.

\section{AGRADECIMENTOS}

À empresa Sakata Seed Sudamerica Ltda pela cessão das sementes. À FAPESP e ao CNPq, pela concessão das bolsas de estudo e suporte financeiro ao projeto.

\section{REFERÊNCIAS}

BRASIL. 2009. Ministério da Agricultura, Pecuária e Abastecimento. Regras para análise de sementes. Secretaria de Defesa Agropecuária. Brasília, DF: Mapa/ACS. 365p.

DUCOURNAU S; FEUTRY A; PLAINCHAULT P; REVOLLON P; VIGOUROUX B; WAGNER MH 2005. Using computer vision to monitor germination time course of sunflower (Helianthus annuus L.) seeds. Seed Science and Technology 33: 329-340.

FESSEL SA; SILVA LJR; GALLI JA; SADER R. 2005. Uso de solução salina $(\mathrm{NaCl})$ no teste de envelhecimento acelerado em sementes de brócolis (Brassica oleracea L. var. italica Plenck). Cientifica 33: 27-34.

FRANZIN SM; MENEZES NL; GARCIA DC; WRASSE CF. 2004. Métodos para avaliação do potencial fisiológico de sementes de alface. Revista Brasileira de Sementes 26: 63-69.

GUIMARÃES JRM; MALAVASI MM; LOPES HM. 1993. Perspectiva da avaliação do vigor de sementes de alface (Lactuca sativa L.) através do comprimento de radícula, utilizando o teste "slant board". Informativo ABRATES 3: 156.

GENEVE RL; KESTER ST. 2001. Evaluation of seedling size following germination using computer-aided analysis of digital images from a flat-bed scanner. HortScience 36: 1117-1120.

HAMPTON JG; TeKRONY DM (ed).1995. 
Handbook of vigour test methods. Zurich: ISTA. $117 \mathrm{p}$.

HOFFMASTER AL; FUJIMURA K ; McDONALD MB; BENNETT MA. 2003. An automated system for vigour testing threeday-old soybean seedlings. Seed Science and Technology 31: 701-713.

JIANHUA Z; McDONALD MB. 1997. The satured salt accelerated aging for smallseeded crops. Seed Science and Technology 25: $123-131$

MAGUIRE JD. 1962. Speed of germination aid in selection and evaluation for seedling and vigour. Crop Science 2: 176-177.

MARCOS FILHO J. 1999. Teste de envelhecimento acelerado. In: KRZYZANOWSKI FC; VIEIRA RD; FRANÇA NETO JB (ed). Vigor de sementes: conceitos e testes. Londrina: ABRATES. p.1-24.

MARCOS FILHO J; BENNETT MA; McDONALD MB; EVANS AF; GRASSBAUGH EM. 2006. Assessment of melon seed vigour by an automated computer imaging system compared to traditional procedures. Seed Science and Technology 34: 485-497.
MARCOS FILHO J; KIKUTI ALP; LIMA LB. 2009. Métodos para avaliação do vigor de sementes de soja, incluindo a análise computadorizada de imagens. Revista Brasileira de Sementes 31: 102-112.

NAKAGAWA J. 1999. Testes de vigor baseados no desempenho das plântulas. In: KRZYZANOWSKI FC; VIEIRA RD; FRANÇA NETO JB (ed). Vigor de sementes: conceitos e testes. Londrina: ABRATES. p. 2.1-2.24.

NASCIMENTO WM; PEREIRA RS. 2007. Testes para avaliação do potencial fisiológico de sementes de alface e sua relação com a germinação sob temperaturas adversas. Revista Brasileira de Sementes 29: 175-179.

OTONI RR; MACDONALD MB. 2005. Moisture and temperature effects on maize and soybean seedlings using the seed vigor imaging system. Seed Technology 27: 243-247.

PEREIRA RS; NASCIMENTO WM. 2003. Testes de vigor na avaliação da qualidade fisiológica de sementes de alface. Informativo Abrates 13: 428.

POWELLAA. 1995. The controlled deterioration test. In: VENTER HA van der (ed). Seed vigour testing seminar. Copenhagen: ISTA. p.73-87.

RODO AB; MARCOS FILHO J. 2003. Accelerated aging and controlled deterioration for the determination of the physiological potential of onion seeds. Scientia Agrícola 60: 465-469

RODO AB; PANOBIANCO M; MARCOS FILHO J. 2000. Metodologia alternativa do teste de envelhecimento acelerado para sementes de cenoura. Scientia Agrícola 57: 289-292.

SAKO Y; McDONALD MB; FUJIMURA K; EVANS AF; BENNETT MA. 2001. A system for automated seed vigour assessment. Seed Science and Technology 29: 625-636.

SEKHARAN S. 2005. Aged soybean seeds - their physiology and vigor assessment. Columbus/ OH/EUA: The Ohio State University. 180p. (Tese doutorado).

TOHIDLOO G; KRUSE M. 2009. Development of an image analysis aided seedling growth test for winter oilseed rape and verification as a vigour test. Seed Science and Technology 37: 98-109. 